$$
\begin{array}{r}
\text { Pontifícia Universidade Católica } \\
\text { do Rio de Janeiro }
\end{array}
$$

Ilitch Vitali Gomes da Silva

\title{
Previsão de Vento para Geração de Energia Elétrica
}

\section{Dissertação de Mestrado}

Dissertação apresentada como requisito parcial para obtenção do título de Mestre pelo Programa de Pós-Graduação em Engenharia Elétrica da PUC-Rio.

Orientador: Prof. Reinaldo Castro Souza Co-orientador: Prof. José Francisco Moreira Pessanha 


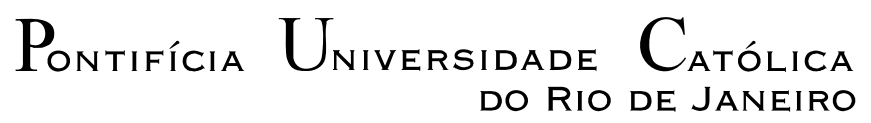

DO RIO DE JANEIRO

Ilitch Vitali Gomes da Silva

\section{Previsão de Vento para Geração de Energia Elétrica}

Dissertação de Mestrado apresentada como requisito parcial para obtenção do grau de Mestre pelo Programa de Pós-Graduação em Engenharia Elétrica do Departamento de Engenharia Elétrica do Centro Técnico Científico da PUC-Rio. Aprovada pela Comissão Examinadora abaixo assinada.

Prof. Reinaldo Castro Souza Orientador Departamento de Engenharia Elétrica - PUC-Rio

Prof. José Francisco Moreira Pessanha Co-orientador UERJ

Profa. Marley Maria Bernardes Rebuzzi Vellasco

Departamento de Engenharia Elétrica - PUC-Rio

Prof. Marcus Vinicius Pereira de Souza Departamento de Engenharia Industrial - PUC-Rio

Prof. José Eugenio Leal Coordenador Setorial do Centro

Técnico Científico - PUC-Rio

Rio de Janeiro, 23 de agosto de 2010 
Todos os direitos reservados. É proibida a reprodução total ou parcial do trabalho sem autorização da Universidade, do autor e do orientador.

\section{Ilitch Vitali Gomes da Silva}

Graduou-se em Ciências Econômicas pela Universidade Federal de Juiz de Fora em 2007. Especialista em Métodos Estatísticos Computacionais pela mesma instituição em 2008.

Ficha Catalográfica

Silva, llitch Vitali Gomes da

A previsão do vento para geração de energia eólica / llitch Vitali Gomes da Silva ; orientador: Reinaldo Castro Souza. -2010.

103 f. : il. (color.) ; $30 \mathrm{~cm}$

Dissertação (mestrado)-Pontifícia Universidade Católica do Rio de Janeiro, Departamento de Engenharia Elétrica, 2010.

Inclui bibliografia

1. Engenharia elétrica, - Teses. 2. Energia eólica. 3. Velocidade do vento. 4. Séries temporais. 5. Box-Jenkins. 6. Neuro-fuzzy. 7. Previsão. I. Souza, Reinaldo Castro. II. II. Pontifícia Universidade Católica do Rio de Janeiro. Departamento de Engenharia Elétrica. III. Título.

CDD: 621.3 


\section{Agradecimentos}

Nesta página, deixo os meus mais verdadeiros sentimentos para as pessoas que fazem parte e continuam presentes em minha vida. Para aqueles que tive oportunidade de conhecer durante os meus estudos.

O meu primeiro agradecimento para o Senhor onipresente e onipotente "Deus", a quem toda honra e glória pertence.

Aos meus queridos pais Alfredo António da Silva e Margarida Alfredo Gomes pela educação, conselhos, perseverança e apoio financeiro. Deles veio a inspiração nos momentos mais difíceis da minha vida. A conquista pessoal deles foi sempre a minha motivação e força para continuar a lutar por os meus objetivos.

Os meus agradecimentos a minha querida esposa Izabela, pelo amor, compreensão, perseverança e sacrifício. Nos momentos difíceis dos meus estudos ela esteve sempre presente e me apoiou incondicionalmente.

Agradeço aos meus irmãos Egberto L. G. da Silva, Laércio A. G. da Silva e Lually da Silva, pela amizade que sempre tiveram para comigo, por aquilo que são e representam na minha vida. Pelo compartilhamento dos momentos bons e baixos da vida.

Os meus agradecimentos ao Prof. Reinaldo Castro Souza, pela orientação, conhecimentos, dedicação e atenção. O meu muito obrigado pela confiança e apoio que a mim prestou quando precisei.

O meu agradecimento ao meu co-orientador e amigo Prof. José Francisco Morreira Pessanha pela orientação, dedicação, atenção, pelos conhecimentos transmitidos na área de energia eólica e sobretudo pela valiosa contribuição prestada nesse trabalho.

Os meus agradecimentos para o Conselho Nacional de Desenvolvimento Científico e Tecnológico - CNPq, a Pontifícia Universidade Católica de Rio de Janeiro pelo apoio financeiro e pela oportunidade de formação.

O meu especial agradecimento ao pessoal do Departamento de Engenharia Elétrica, na pessoa do Coordenador do Curso de Pós-Graduação Professor Ricardo Prado, Alcina Portes e Ana Luiza. 
Os meus agradecimentos para os colegas e amigos da PUC, Schaiane Nogueira Ouverney Barroso e Paulo Roberto Bastos Maia pelo compartilhamento de conhecimentos e amizade que fizemos ao longo do mestrado.

Ao meu amigo Jonas Ferreira Pereira pela amizade, pelos conselhos, brincadeiras, e principalmente pelo apoio que deu quando mais precisei.

O meu profundo e sentido agradecimento a todas as pessoas que contribuíram direta e indiretamente para a concretização desta dissertação, estimulando-me intelectual e emocionalmente.

Obrigado Deus por acreditar em mim. 


\section{Resumo}

Silva, Ilitch Vitali Gomes da; Souza, Reinaldo Castro; Pessanha, José Francisco Moreira. Previsão de Vento para Geração de Energia Elétrica. Rio de Janeiro, 2010, 103p. Dissertação de Mestrado-Departamento de Engenharia Elétrica, Pontifícia Universidade Católica de Rio de Janeiro.

A energia eólica é uma das alternativas mais promissoras para geração de energia elétrica, pois assegura a diversidade e segurança no fornecimento de energia e atende à necessidade premente de reduzir os níveis de emissão de gases poluentes. Na operação de sistemas elétricos com forte presença de geração eólica é fundamental prever com pelo menos um dia de antecedência os valores futuros (pelo menos horários) da velocidade do vento, pois assim pode-se avaliar a disponibilidade de energia para o próximo dia, uma informação útil no despacho das unidades geradoras e no controle do sistema elétrico. A proposta dessa dissertação objetiva especificamente desenvolver modelos de previsão de curto prazo da velocidade do vento, baseado em técnicas de inteligência artificial, modelo da rede neural artificial e neuro-fuzzy adaptativa (ANFIS) e um modelo Estatístico composto por um modelo de regressão harmônica e Box-Jenkins. Para aplicação da metodologia considerou-se o município de São João do Cariri (Estado de Paraíba), onde está localizada uma das estações de referência do projeto SONDA (Sistema Nacional de Dados Ambientais para o setor de energia). O desempenho dos modelos rede neural, neuro-fuzzy (ANFIS) e modelo Estatístico são comparados nas previsões de 6 horas, 12 horas, $18 \mathrm{~h}$ e 24 horas a frente. Os resultados obtidos mostram o melhor desempenho da modelagem ANFIS e encorajam novos estudos no tema.

\section{Palavras chave}

Energia eólica; velocidade do vento; séries temporais; Box-Jenkins; neuro-fuzzy; previsão. 


\section{Abstract}

Silva, Ilitch Vitali Gomes da; Souza, Reinaldo Castro (Advisor); Pessanha, Jose Francisco Morreira (Co-advisor). The Wind Forecast for Wind Power Generation. Rio de Janeiro, 2010, 103p.MSc. Dissertation - Departamento de Engenharia Elétrica, Pontifícia Universidade Católica de Rio de Janeiro.

Wind power is one of the most promising options for power generation. It ensures the diversity and security of energy supply and meets the pressing need to reduce the levels of emission of polluting gases. In the operation of electrical systems with a strong presence of wind generation, it is essential to provide at least one day in advance the future values (at least hourly) of wind speed, so that we can assess the availability of energy for the next day, a useful information in the order of the generating units and electrical control system. The purpose of this dissertation aims to develop models specifically to develop models to forecast short-term wind speed, based on artificial intelligence techniques, artificial neural network model and adaptive neuro-fuzzy Systems (ANFIS) and a statistical model composed of a harmonic regression model and BoxJenkins. For application of the methodology, the city of São João do Cariri (State of Paraíba), where a reference station of SONDA project (National Environmental Data for the energy sector) is located, was considered.To apply the methodology was considered the city of the ray tracing model (State of Paraíba), which is located a station reference design (National Environmental Data for the energy sector). The performance of artificial neural network model and adaptive neuro-fuzzy Systems (ANFIS) and a statistical model are compared mixed forecasts of 6 hours, 12 hours, 18hours and 24 hours ahead. The results show the best performance of the ANFIS model and encourage further studies on the subject.

\section{Keywords}

Wind power; wind speed; time series analysis; Box-Jenkins neuro-fuzzy; forecast. 


\section{Sumário}

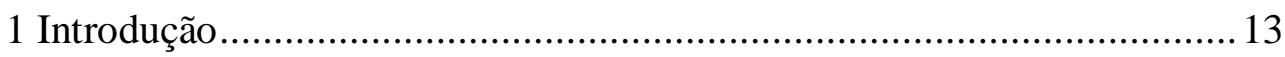

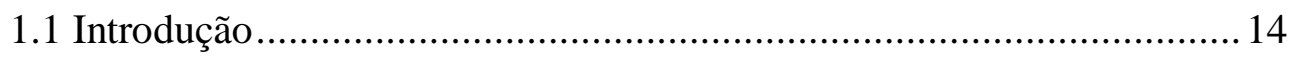

1.1.2 Panorama da Energia Eólica no Mundo ......................................... 16

1.2 Panorama da Energia Elétrica no Brasil........................................... 18

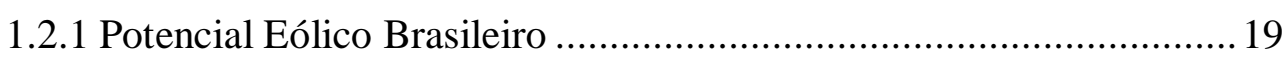

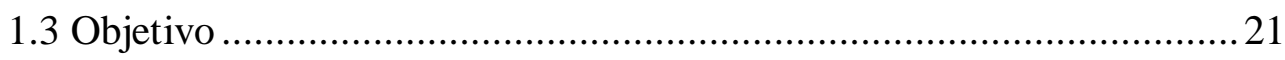

1.4 Estrutura da Dissertação ............................................................ 22

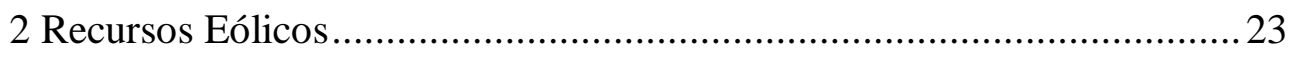

2.1 Integração da Energia Eólica .........................................................25

2.2 Produção da Energia Eólica...........................................................25

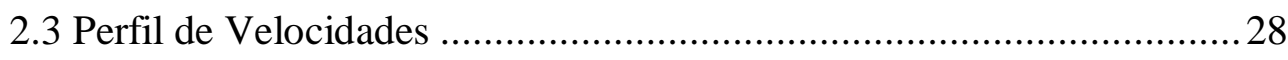

2.4 Modelos de Previsão para Geração de Energia Eólica......................... 29

2.4.1 Modelo Físico ou Atmosférico .....................................................29

2.4.2 Modelos Estatísticos e de Inteligência Computacional ..................... 30

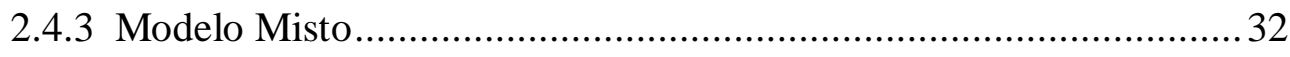

3 Métodos de Previsão de Séries Temporais ............................................... 35

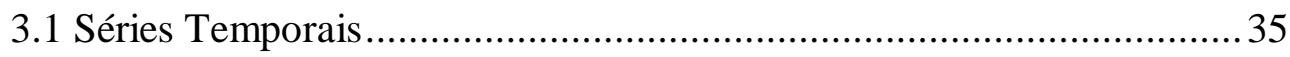

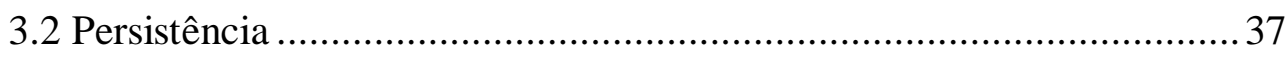

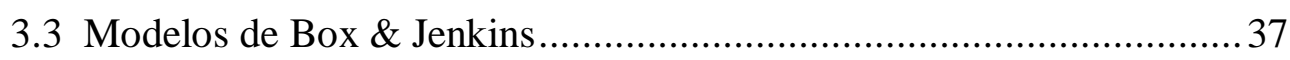

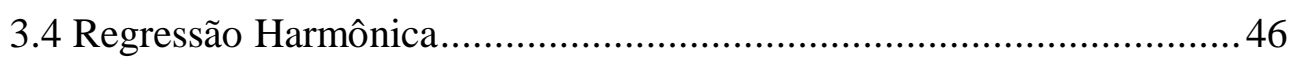

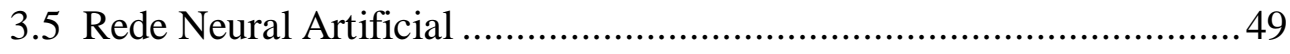

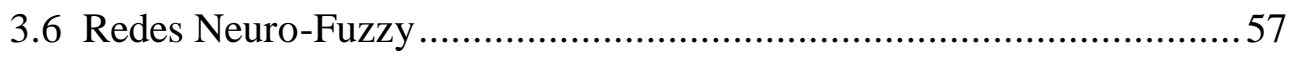

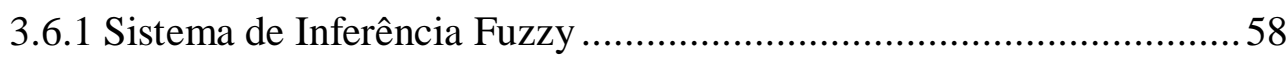

3.6.2 Sistema Neuro-Fuzzy Adaptativo (ANFIS) ..................................63

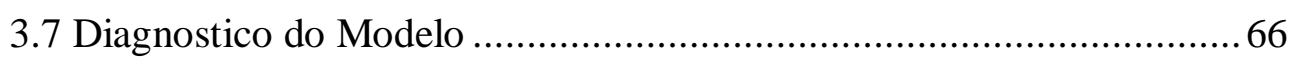

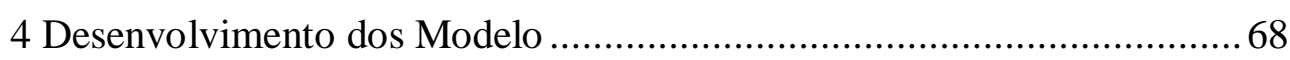

4.1 Análise Exploratória da Série de Velocidade do Vento. ......................69

4.2 Modelo Estatístico de Previsão ........................................................73

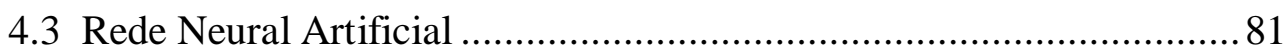

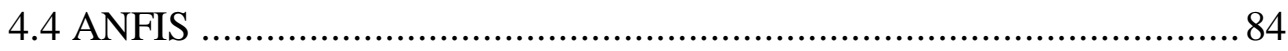


5 Comparação dos Modelos ..................................................................93

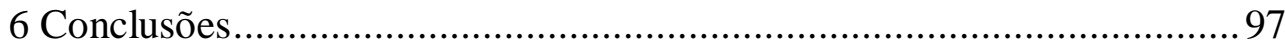

7 Referência Bibliográfica ....................................................................... 100 


\section{Lista de Figuras}

Figura 1-Comparação da produção de energia nos países da OECD. .................. 15

Figura 2-Capacidade instalada de energia eólica no mundo ............................... 18

Figura 3- Mapa da velocidade média anual do vento $(\mathrm{m} / \mathrm{s})$ a 50 metros. ............. 20

Figura 4-Formação dos ventos devido ao deslocamento das massas de ar...........24

Figura 5. Curva de potência típica de uma turbina eólica.................................2 27

Figura 6-Neurônio artificial de McCulloch \& Pitts .......................................... 49

Figura 7-Rede neural com três camadas ..................................................... 51

Figura 8- Montagem do conjunto de treinamento .........................................5 53

Figura 9-Rede neural com três camadas ........................................................5 54

Figura 10-Conjuntos fuzzy e funções de pertinência para a variável temperatura 59

Figura 11-Sistema de inferência fuzzy ou FIS (Pacheco \&Vellasco, 2007) .........60

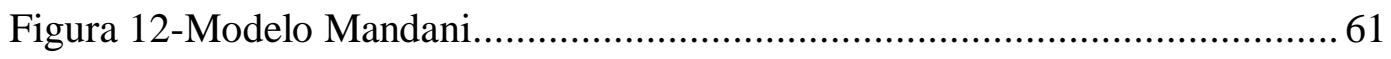

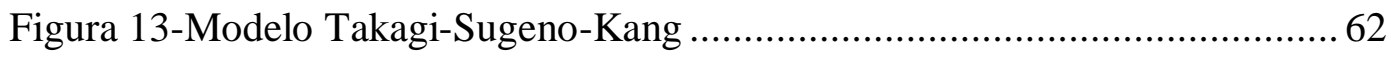

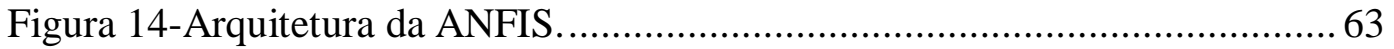

Figura 15-Município de São João do Cariri. ....................................................... 68

Figura 16-Atlas do potencial eólico brasileiro $(\mathrm{m} / \mathrm{s})$.......................................6 69

Figura 17- Série horária de velocidade do vento..........................................69

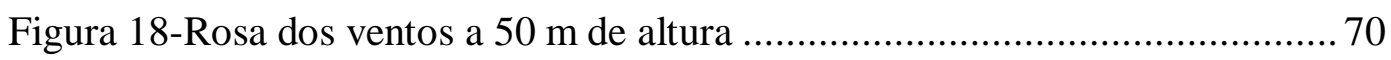

Figura 19-Médias horárias da velocidade do vento $(\mathrm{m} / \mathrm{s})$.............................. 71

Figura 20-Médias mensais da velocidade do vento $(\mathrm{m} / \mathrm{s})$................................ 71

Figura 21- Histograma da velocidade do vento a $50 \mathrm{~m}$ de altura...................... 72

Figura 22-Q-QPlot da da velocidade do vento a 50 metros de altura..................72

Figura 23-FAC de série horária da velocidade do vento. ..................................73

Figura 24-Periodograma da velocidade do vento .......................................... 74

Figura-25-Série dos resíduos da regressão harmônica...................................... 76

Figura 26-Histograma dos resíduos da regressão harmônica .............................77

Figura 27-Q-QPlot dos resíduos da regressão harmônica................................... 77

Figura 28-Resíduo FAC e FACP .......................................................... 78

Figura 29-Previsão da velocidade do vento até 24 horas à frente ....................... 81

Figura 30-Gráfico de treinamento em relação ao observado .............................. 82 
Figura 31-Desempenho da rede em relação aos dados observados..................... 83

Figura 32-Previsão do modelo rede neural 24 horas. ....................................... 83

Figura 33-Série transformada da velocidade do vento ..................................... 84

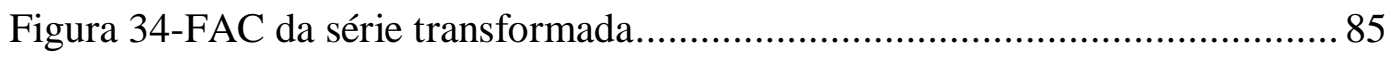

Figura 35-Estrutura do modelo ANFIS.................................................. 89

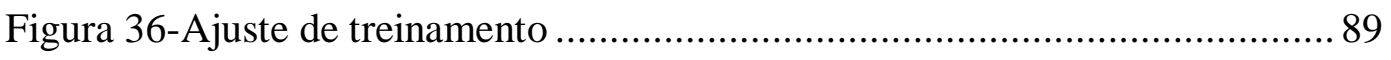

Figura 37-Histograma de dispersão entre observado e estimado. ........................ 90

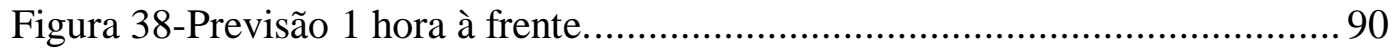

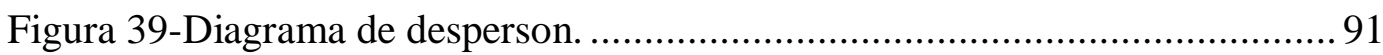

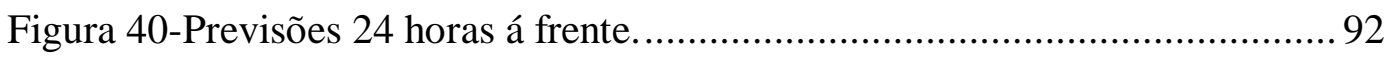

Figura 41-Gráfico de previsão do modelo naive. .............................................93

Figura 42-Comparação das previsões. ........................................................ 96 


\section{Lista de Tabela}

Tabela 1-Consumo de energia nos países da OECD. ....................................... 15

Tabela 2-Potencial acumulada de energia eólica ao final de anos...................... 17

Tabela 3-Resultado do leilão de energia eólica de reserva. ................................ 20

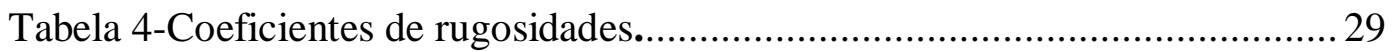

Tabela 5-Modelos de previsão utilizados na Europa.......................................... 34

Tabela 6-Características principais dos modelos $\operatorname{AR}(p), \operatorname{MA}(q)$ e ARMA (p,q).. 42

Tabela 7-Estatísticas de velocidade do vento................................................... 70

Tabela 8-Teste de Normalidade Kolmogorov-Smirnov.................................. 73

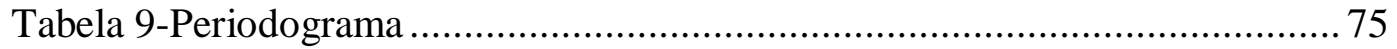

Tabela 10-Resultado de MAPE dos ws estimados. ........................................... 76

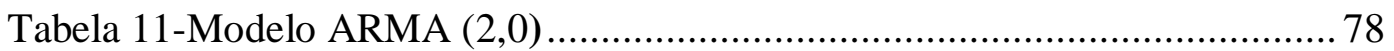

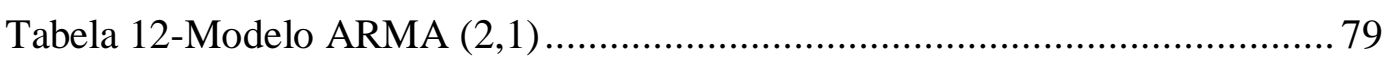

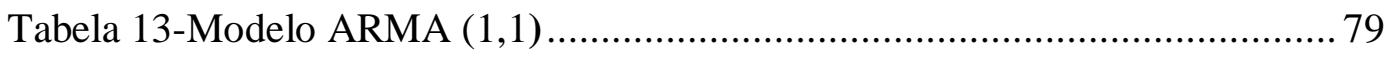

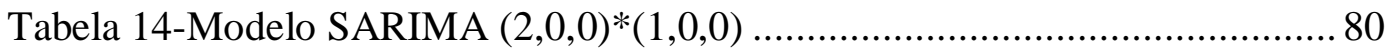

Tabela 15-Resultado de previsão dentro da amostra do modelo estatístico.......... 81

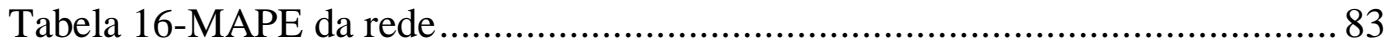

Tabela 17-Resultado de previsão dentro da amostra do modelo rede neural........8 84

Tabela 18-Configurações avaliadas para a rede ANFIS ................................ 87

Tabela 19-Simulação da rede com 700 épocas.............................................. 88

Tabela 20-Resultado de previsão dentro da amostra do modelo ANFIS............. 91

Tabela 21-Comparação dos modelos de previsão dentro da amostra................... 94

Tabela 22-Comparação dos modelos de previsão para 6 horas........................... 94

Tabela 23-Comparação dos modelos de previsão para 12 horas......................... 94

Tabela 24-Comparação dos modelos de previsão para 18 horas.........................95

Tabela 25-Comparação dos modelos de previsão para 24 horas......................... 95 